\title{
Production of hollow hemisphere shells by pure Kirkendall porosity formation in Au/Ag system
}

\author{
Györgyi Glodán, ${ }^{1}$ Csaba Cserháti, ${ }^{1}$ Imre Beszeda, ${ }^{2}$ and Dezső L. Beke ${ }^{1, a)}$ \\ ${ }^{1}$ Department of Solid State Physics, University of Debrecen, 4010 Debrecen, P.O. Box 2, Hungary \\ ${ }^{2}$ Department of Physics, University College of Nyíregyháza, 4400 Nyíregyháza, Sóstói u. 9-11., Hungary
}

(Received 20 May 2010; accepted 27 August 2010; published online 17 September 2010)

\begin{abstract}
Interdiffusion has been studied in $\mathrm{Ag} / \mathrm{Au}$ hemispherical core-shell structures on sapphire substrate. In isothermal heat treatments first a relatively fast growth of nanovoids was observed, which was followed by a slower shrinkage process. The void formation is interpreted by pure Kirkendall-porosity formation since Ag-50\% Au solid solution has been formed in the shell. In contrary, in all previous publications on hollow nanoshell formation a chemical reaction took place and the shell consisted of the reaction product (i.e., of sulphide or oxide). Furthermore, in these cases the shrinkage was observed at temperatures higher than the formation temperature. (C) 2010 American Institute of Physics. [doi:10.1063/1.3490675]
\end{abstract}

It is well-known that voids can be formed during interdiffusion in binary systems (Kirkendall porosity formation ${ }^{1}$ or Frenkel effect $\left.{ }^{2,3}\right)$. The origin of this effect is the resultant vacancy flow (caused by the inequality of the intrinsic atomic fluxes in the lattice frame of reference) oriented toward the faster component. In fact this resultant volume flow is responsible for the development of stress free strain in the diffusion zone: one side tends to shrink and the other one to expand. Additional stress free strains can also develop due to the difference of the atomic volumes and/or to the formation of intermetallic compounds in which the specific volumes of the constituents can be considerably different than in the parent phases. ${ }^{4}$

The partial or full relaxation of these diffusional stresses can lead to the well-known Kirkendall shift: in case of vacancy mechanism, if the efficiency of the vacancy sources and sinks (at dislocations) is high enough, atomic planes will be removed/inserted at the corresponding fast/slow component of the diffusion couple. If this process is fast and complete then the stresses will be relaxed and the pure Darkenregime is realized. Thus the overall intermixing in the laboratory frame of reference is controlled by the larger diffusion coefficient. In real systems, however, more complex time evolution is expected. It can happen that the restricted efficiency of vacancy sinks leads to vacancy super saturation and porosity formation on the side of the faster component. These pores then can also sink vacancies and thus this will be a competing process with the vacancy annihilation at dislocations, i.e., with the Kirkendall shift. ${ }^{2}$ Furthermore, the additional diffusional stresses can enhance or suppress the porosity formation. This complex interplay of the above effects is a delicate theoretical problem and it is expected that simple analytical solutions can work only at very special simple cases. In addition, if the sample geometry is closed (cylindrical, spherical samples), since the stress free strain fields are long range fields, the stress development and relaxation will certainly depend on the shape and size of the sample and for example a radius dependence of the processes are also expected. ${ }^{4,5}$ In extreme circumstances even a switching between the Darken and Nernst-Planck limits can be

${ }^{a)}$ Electronic mail: dlbeke@tigris.klte.hu. observed. ${ }^{5}$ In the latter regime the intermixing is controlled by the diffusion of the slower component. ${ }^{4,5}$

Precursor experiments of hollow nanoshell formations were carried out by Aldinger ${ }^{6}$ and by the Geguzin group. ${ }^{2,7}$ Aldinger observed hollow shells of BeNi alloys formed from $\mathrm{Be} / \mathrm{Ni}$ core/shell microparticles. ${ }^{6}$ Hollow intermetallic shell formation in $\mathrm{Ni}$ coated $\mathrm{Cd}$ wires was described and interpreted by a large change (about $8 \%$ ) of the molar volume during the intermetallic formation., ${ }^{2,7}$

While the above hollow shells or cylinders were formed in diffusion samples of micrometer dimensions, since 2004 papers on hollow nanoshell formation were published, ${ }^{8-14}$ in which metallic spheres were reacted by oxygen and sulfur, and oxide or sulphide nanoshells were obtained. In these works the formation mechanism of hollow spheres was described as an analogous phenomenon to the Kirkendall porosity formation. It was also observed ${ }^{14}$ that the growth of the thickness of the oxide shell at fixed temperature for $\mathrm{Cu}$, $\mathrm{Zn}$, and $\mathrm{Al}$ oxides had a saturation character: after an initial fast growth it reached a saturation.

The above nanohollow structures should be unstable due to the Gibbs-Thomson effect: ${ }^{15,16}$ the vacancy concentration on the inner boundary of the nanoshell should be higher than that on the external boundary. Thus an outward vacancy flux is created which leads to shrinkage. In Refs. 6, 17, and 18 it was argued that, contrary to the shrinking of singlecomponent shell, shrinking of a binary compound shell should be effected by the inverse Kirkendall effect too: the outward vacancy flux leads to segregation of the faster component near the inner boundary. The corresponding concentration gradient can suppress the vacancy flux. Therefore, the shrinkage rate of the compound shell can be slowed down. The shrinkage of compound shells was experimentally verified by Nakamura et al.: ${ }^{19}$ they observed shrinkage of Ni and $\mathrm{Cu}$ oxide particles at higher temperatures than at which they were formed. In addition it was shown from model calculations $^{20}$ that the Gibbs-Thomson effect, leading to shrinkage of "ready" compound shells, should be important at the formation stage as well and it may even suppress the nanoshell formation.

Up to now the hollow nanoshell formation, both experimentally and theoretically, was considered only for cases 
when a reaction product forms the shell and almost exclusively the two primary effects (the vacancy flow oriented toward the center as well as segregation) was treated. In these cases, however, the stress development caused by the abrupt change in the specific volumes during compound formation should be considerable and it is not clear yet what is its role in the void formation. Thus, as a first step, it is desirable to carry out experiments in which the main effect is only the oriented vacancy flow and other stress free strains can be neglected. Very recently theoretical papers ${ }^{3,21-23}$ addressed exactly the problem of nanoshell formation in binary systems with full or very wide solubility range (but again not considering stress effects). They have shown that first there was a relatively fast growing stage and, after a maximum in porosity, a slower shrinkage process took place at fixed temperature.

In this communication we present experimental result on hollow hemisphere formation in almost ideal $\mathrm{Au} / \mathrm{Ag}$ binary system, where the void formation is almost exclusively related to the unequal intrinsic atomic fluxes and other stress effect can be neglected. Thus pure Kirkendall porosity formation can be observed. It is interesting to note that (using vacancy formation energies from Refs. 24 and 25, and taking the vacancy formation entropy equal to the Boltzmann constant) in pure $\mathrm{Ag}$ and $\mathrm{Au}$ the equilibrium vacancy concentration at $743 \mathrm{~K}$ (used in our experiment) is about $3.4 \times 10^{-4}$ and $5.2 \times 10^{-5}$ at. $\%$, respectively, while this value in the $\mathrm{Ag}(50) \mathrm{Au}(50)$ solid solution is about $1.3 \times 10^{-4}$ at. $\%$.

Continuous (of about $10 \mathrm{~nm}$ thick) $\mathrm{Ag}$ layers were evaporated onto the polished surface of $\mathrm{Al}_{2} \mathrm{O}_{3}\langle 0001\rangle$ substrate of $5 \times 5 \mathrm{~mm}^{2}$ dimension. For the production of $\mathrm{Ag}$ hemispheres the method described in Ref. 26 was followed: during annealing $(6 \mathrm{~h}$ at $973 \mathrm{~K}$ in reducing atmosphere of $5 \% \mathrm{H}+95 \%$ Ar mixture) the continuous film gradually disintegrated and separated beads were formed having shapes of hemispheres. The diameters of the hemispheres varied between 20 and $50 \mathrm{~nm}$, i.e., the average diameter was about $35 \mathrm{~nm}$. As a second step a thin Au film (about $10 \mathrm{~nm}$ ) was evaporated on the top of $\mathrm{Ag}$ beads as well as on the clean surface of the substrate. The average composition of the core-shell structures formed was approximately 50\%-50\%. The base vacuum during the evaporation was $10^{-6}$ mbar.

The specimens were annealed in reducing atmosphere at $723 \mathrm{~K}$ and $743 \mathrm{~K}$ for $10,15,20,30,60$, and $180 \mathrm{~min}$. The heat treatment was performed in dynamic reducing gas flow.

After annealing the beads were removed from the $\mathrm{Al}_{2} \mathrm{O}_{3}$ substrate by tearing off the dried film formed by dropping collodion solution onto the surface. The dried film was placed onto a transmission electron microscope (TEM) grid covered with amorphous carbon layer and after the dissolution of the carrier layer the back sides of the beads were investigated by JEOL 2000FX-II TEM/EDX system at 200 $\mathrm{keV}$ on plan view.

The area of the holes and beads were measured by standard image processing method. After some noise reduction, the edges of the beads and pores were detected at several points. The points were than connected with straight sections and the area of the internal part had been calculated. Using the scale mark of the TEM pictures we could calibrate the distances on the images and calculate the areas in units of square nanometer.
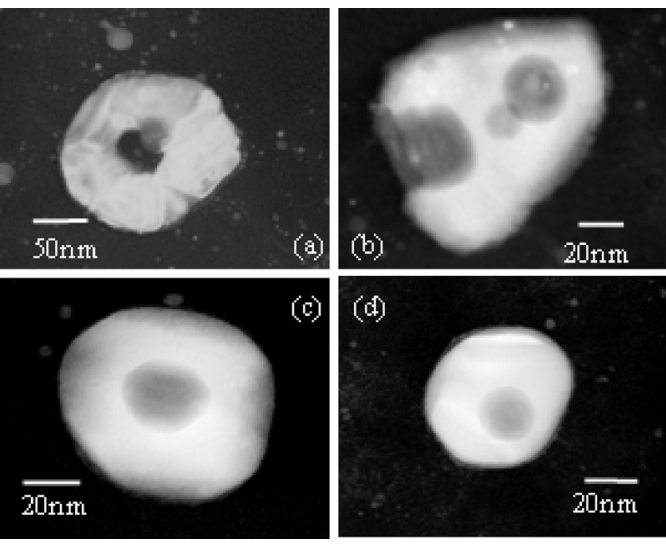

FIG. 1. TEM picture of AgAu beads formed at $743 \mathrm{~K}$ (a) $10 \mathrm{~min}$, (b) 20 min, (c) $30 \mathrm{~min}$, and (d) $60 \mathrm{~min}$

Figure 1 shows the obtained porous hemispheres at 743 $\mathrm{K}$. It can be seen that there is porosity inside the beads. Its relative volume (as compared to the volume of the particle) first increases, reaches a maximum (at about $30 \mathrm{~min}$ ) and then gradually decreases. In accordance with simulations, ${ }^{3}$ in the growing stage of pores there are usually more than one pore inside the particles [Fig. 1(b)]. At the lower temperature $(723 \mathrm{~K})$ we observed more individual smaller sized pores even at the maximum. Figure 1(a) illustrates also that the interdiffusion process is faster along the grain-boundaries (several grains can be identified). In general it can be concluded that the voids formed can be symmetric, asymmetric and one bead can contain a few voids as well.

It is plausible to plot the ratio of the area of the pore, $a$, and particle, $A$, versus the time, similarly as the volume ratio was plotted from the simulations in Ref. 23. This plotting is advantageous, because in our case the initial size of the beads showed a distribution and because the area ratio clearly mimics the volume ratio.

Figure 2 shows this function which is quite similar to Fig. 7 in Ref. 3 and to Fig. 5 in Ref. 23. The size of the voids depends on the temperature: at lower temperature the average area is smaller. At $743 \mathrm{~K}$ after $3 \mathrm{~h}$ heat treatment the voids completely disappeared and at $723 \mathrm{~K}$ the shrinking process was slower. It can also be seen that the formation stage is much shorter than the shrinking one.

It is important noting that in experiments on shrinking of oxide particles ${ }^{19}$ the shrinkage was observed at higher temperatures than the formation temperature of nanoshells. This was interpreted by the idea ${ }^{16}$ that rate of shrinking is controlled by the slower diffusing species (Nernst-Planck steady state limit). On the other hand it was shown from kinetic

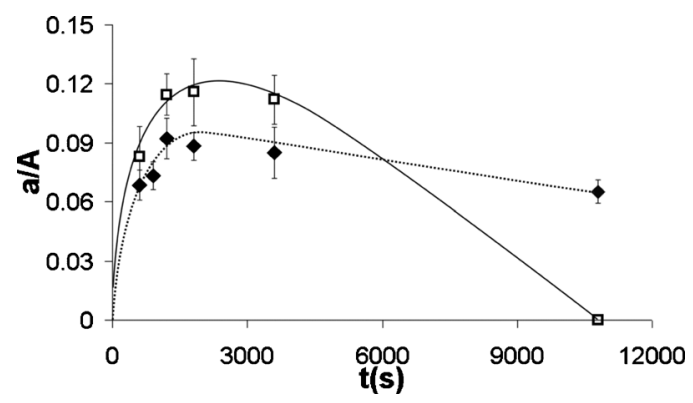

FIG. 2. Relative area of the pores as a function of the annealing time at $743 \mathrm{~K}$ (open squares) and at $723 \mathrm{~K}$ (full marks). 


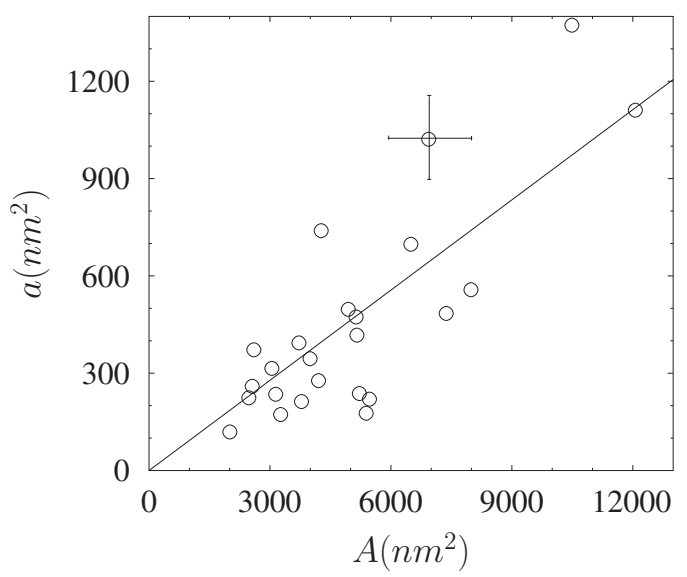

FIG. 3. Pore area vs particle area at $723 \mathrm{~K}$

Monte Carlo calculations ${ }^{22}$ that the shrinkage started before the complete intermixing has been reached and the shrinking rate was only tree times slower than the formation rate at fixed temperature. The authors of Ref. 22 expressed their doubts whether the shrinkage is a steady state process and controlled by the diffusion of the slower component. Our results seems to support these arguments, but further experiments and modeling is necessary for different diffusion couples to arrive at an equivocal conclusion (in $\mathrm{Ag}-\mathrm{Au}$ system, as compared to oxides, the difference between the intrinsic diffusion coefficients is very moderate and the Darken and Nernst-Plank limits should be much closer to each other).

Since in our ensemble a distribution of beads is present (the diameter of particles in the initial core/shell structure have been varied between 30 and $90 \mathrm{~nm}$ ), it is possible to plot the pore area versus the particle area. Figure 3 shows this function $723 \mathrm{~K}$ at the time belonging to the maxima in Fig. 2. It can be seen that, in spite of the relatively large experimental scatter, it can be fitted by a straight line. The slopes of these linear functions are similar at the two temperatures investigated $(0.12$ and 0.09 at $743 \mathrm{~K}$ and $723 \mathrm{~K}$, respectively).

We have shown that nanovoids have been formed in Ag$50 \% \mathrm{Au}$ core/shell hemispheres, i.e., a pure Kirkendallporosity formation took place. Following the kinetics of the process by TEM at 723 and $743 \mathrm{~K}$ first a relatively fast growth of the holes was observed which was followed by a slower shrinkage process, in accordance with the results of simulations and analytical model-calculations. In contrary to our investigations, in which $\mathrm{Ag} / \mathrm{Au}$ solid solution shells formed, in all previous publications on hollow nanoshell for- mation a chemical reaction took place (accompanied by serious stress free strain accumulation) during the formation of nanoholes and the shell consisted of the reaction product (i.e., of sulfide or oxide). Furthermore, in the only one experimental work ${ }^{19}$ on the shrinkage kinetics (of oxide hollow particles), the sintering of the voids was observed at higher temperatures than the formation temperature of nanoshells.

This work was supported by Grant No. CK 80126 of the Hungarian Scientific Research Fund and by the TÁMOP under Project No. 4.2.1-08/1-2008-003.

${ }^{1}$ J. Philibert, Atom Movement, Diffusion and Mass Transport in Solids (Le Edition de Physique, Les Ulis, 1991), p. 220

${ }^{2}$ Y. E. Geguzin, Diffusion Zone (Nauka, Moscow, 1979).

${ }^{3}$ A. M. Gusak and T. V. Zaporozhets, J. Phys.: Condens. Matter 21, 415303 (2009)

${ }^{4}$ D. L. Beke, I. A. Szabo, Z. Erdelyi, and G. Opposits, Mater. Sci. Eng., A 387-389, 4 (2004).

${ }^{5}$ G. Schmitz, C. B. Ene, and C. Nowak, Acta Mater. 57, 2673 (2009).

${ }^{6}$ F. Aldinger, Acta Metall. 22, 923 (1974).

${ }^{7}$ Y. E. Geguzin, Y. Klinchuk, I. Yu, and L. N. Paritskaya, Fiz. Met. Metalloved. 43, 602 (1977)

${ }^{8}$ Y. Yin, R. M. Rioux, C. K. Erdonmez, S. Hughes, G. A. Somorjai, and A. P. Alivisatos, Science 304, 711 (2004).

${ }^{9}$ Y. Yin, C. K. Erdonmez, A. Cabot, S. Hughes, and A. P. Alivisatos, Adv. Funct. Mater. 16, 1389 (2006).

${ }^{10}$ C. M. Wang, D. R. Baer, L. E. Thomas, J. E. Amonette, J. Antony, Y. Qiang, and G. Duscher, J. Appl. Phys. 98, 094308 (2005).

${ }^{11}$ R. Nakamura, J. G. Lee, D. Tokozakura, H. Mori, and H. Nakajima, Mater. Lett. 61, 1060 (2007).

${ }^{12}$ R. Nakamura, D. Tokozakura, H. Nakajima, J. G. Lee, and H. Mori, J. Appl. Phys. 101, 074303 (2007).

${ }^{13}$ R. Nakamura, J. G. Lee, H. Mori, and H. Nakajima, Philos. Mag. 88, 257 (2008).

${ }^{14}$ H. Nakajima and R. Nakamura, J. Nano Res. 7, 1 (2009).

${ }^{15}$ K. N. Tu and U. Gosele, Appl. Phys. Lett. 86, 093111 (2005).

${ }^{16}$ A. M. Gusak, T. V. Zaporozhets, K. N. Tu, and U. Gosele, Philos. Mag. 85, 4445 (2005).

${ }^{17}$ I. V. Belova and G. E. Murch, J. Phase Equilib. Diffus. 26, 430 (2005).

${ }^{18}$ A. V. Evteev, E. V. Levchenko, I. V. Belova, and G. E. Murch, Philos. Mag. 87, 3787 (2007).

${ }^{19}$ R. Nakamura, D. Tokozakura, J. G. Lee, H. Mori, and H. Nakajima, Acta Mater. 56, 5276 (2008).

${ }^{20}$ A. M. Gusak and K. N. Tu, Acta Mater. 57, 3367 (2009).

${ }^{21}$ A. V. Evteev, E. V. Levchenko, I. V. Belova, and G. E. Murch, J. Nano Res. 7, 11 (2009).

${ }^{22}$ A. V. Evteev, E. V. Levchenko, I. V. Belova, and G. E. Murch, Philos. Mag. 88, 1525 (2008).

${ }^{23}$ G. E. Murch, A. V. Evteev, E. V. Levchenko, and I. V. Belova, Diffus Fundam. 42, 1 (2009).

${ }^{24}$ R. J. Borg and G. J. Dienes, An Introduction to Solid State Diffusion (Academic, London, 1988).

${ }^{25}$ D. L. Beke, I. Gödény, F. J. Kedves, and G. Erdélyi, J. Phys. Chem. Solids 40, 543 (1979).

${ }^{26}$ I. Beszeda, I. A. Szabó, and E. G. Gontier-Moya, Appl. Phys. (Berlin) A78, 1079 (2004). 